\section{SOI: 1.1 TAS DOI: 10.15863 /TAS \\ International Scientific Journal Theoretical \& Applied Science}

Gauhar Sarsenbaevna Borankulova

Ph.D.,

TarSU M.H.Dulati, Kazakhstan

b.gau@mail.ru

Aigul Turyszhanovna Tungatarova

Ph.D.,

Department of Informatics,

TarSU M.H.Dulati, Kazakhstan

at.tu@mail.ru

SECTION 4. Computer science, computer engineering and automation.

\title{
AUTOMATION EXPERTISE TRAINING PROGRAM DISCIPLINE
}

Abstract: In the article the questions of automation of examination of curriculum subjects. The proposed examination is based on a comparison database created for each discipline individually.

Key words: curriculum, automation expertise, keywords, keyword database

Language: Russian

Citation: Borankulova GS, Tungatarova AT (2015) AUTOMATION EXPERTISE TRAINING PROGRAM DISCIPLINE. ISJ Theoretical \& Applied Science 05 (25): 135-139.

Soi: http://s-o-i.org/1.1/TAS*05(25)25 Doi: crossef http://dx.doi.org/10.15863/TAS.2015.05.25.25

\section{АВТОМАТИЗАЦИЯ ЭКСПЕРТИЗЫ ПРОГРАММЫ ОБУЧЕНИЯ ДИСЦИПЛИНЫ}

Аннотация: В статье рассматриваются вопросы автоматизации экспертизы программы обучения дисциилины. Предложенная экспертиза основана на сравнении базы ключевых слов, созданной для каждой дисииплины индивидуально.

Ключевые слова: программа обучения, автоматизация, экспертиза, ключевые слова, база ключевых слов.

Эффективность обучения в системе высшего образования во многом зависит от более высокого уровня организации учебного процесса, внедрения эффективных форм и методов обучения, широкого применения в учебном процессе современных учебных пособий и инновационных технологий.

Не маловажную роль в повышении качества образования занимает учебно-методическая база как кафедры, так и вуза в общем, одной из составляющих которой является программа обучения дисциплины.

При создании программы обучения дисциплины (ПОД) преподаватели придерживаются требований государственного стандарта образования, предъявляемых к составлению программы обучения дисциплины. Оформление программы обучения дисциплины проводится по утвержденному в вузе образцу. Следовательно, проблем по оценке соответствия оформления утвержденному требованию не возникает. Проблема возникает при проведении экспертизы соответствия содержания теоретической части программы обучения требованиям стандарта.

Экспертиза программы обучения дисциплины проводится вручную. Возникает необходимость автоматизировать процесс экспертизы программы. Основной идеей процесса автоматизации экспертизы программы обучения дисииплины является создание базы ключевых слов для каждой дисциплины. Сравнение ключевых слов по каждой дисциплине позволит вычислить процент совпадения ключевых слов. При превышении процентного соотношения $40 \%$, проходящая экспертизу дисциплина считается дублирующей предыдущую дисциплину, то есть признается не соответствующей требованиям стандарта, в результате чего данная программа обучения дисциплины отправляется на переработку.

Согласно «Положение о разработке учебнометодических комплексов специальностей и дисциплин» ответственность за составление ПОД несет преподаватель или коллектив преподавателей кафедры, являющиеся разработчиками программы обучения. При составлении ПОД строго учитываются требования государственного обязательного стандарта образования по специальностям и требования «Положения об учебно-методическом комплексе по дисциплине», принятом и утвержденном учебным заведением. Все готовые 
УП по преподаваемым на кафедре дисциплинам оформляются в соответствии с требованиями Положения и обсуждаются на кафедры. Во многих вузах экспертиза программы обучения на этом заканчивается.

После того, как формирование программы обучения дисциплины подошло к концу, необходимо провести на кафедре коллективную экспертизу ключевых слов с преподавателями, читающими данную дисциплину для специальности.

При проведении экспертизы ключевых слов опираются на основные термины и определения из стандарта терминов Республики Казахстан (СТ РК 34005-2002). Настоящий стандарт устанавливает основные термины и определения в области информационных технологий.

Термины, установленные настоящим стандартом, обязательны для применения во всех видах документации и литературе по информационным технологиям. Установленные в стандарте термины расположены в систематизированном порядке, отражающем систему понятий в области информационных технологий. Для каждого понятия установлен один стандартизованный термин.

При необходимости, разрешается уточнять приведенные определения, вводя дополнительные признаки, раскрывающие значения терминов, без искажения смысла определения.

Формирование базы ключевых слов является необходимым условием при проведении экспертизы, поскольку именно ключевые слова в точности передают основное содержание теоретического материала дисциплины.

Нужно учесть, что в словаре терминов могут встретиться слова синонимы, то есть слова, разные по написанию, но имеющие один и тот же смысл. Следовательно, если при создании программы не учесть наличие слов синонимов, два синонима при сравнении будут восприняты как два разных по смыслу слова, а значит, программа выдаст неверный результат экспертизы. Поэтому на базе ключевых слов формируется словарь синонимов предметной области дисциплины.

Имея базу ключевых слов и словарь синонимов можно вводить данные в программу для проведения экспертизы программы обучения дисциплины на соответствие.

Готовые программы обучения дисциплин в электронном варианте должны быть переданы для проведения экспертизы на соответствие в специально организованное структурное подразделение, ответственное за проведение экспертизы программ обучения. К электронной версии программы обучения дисциплины прилагается файл ключевых слов и словарь синонимов. Электронная версия программы обучения дисциплины помещается в специально созданную папку, которая привязана к программе проведения экспертизы программы обучения дисциплины. В случае необходимости пользователь может непосредственно в окне программы через клавишу «Сформировать каталог для УП» открыть и просмотреть электронную версию учебной программы.

В качестве входной информации служит файл ключевых слов и словарь синонимов. Словарь синонимов заносится в базу, просмотр которой осуществляется через кнопку «Синонимы» в окне основной программы. Причем, базу слов синонимов можно пополнять или удалять из нее ненужные слова синонимы в ходе выполнения программы.

Файл ключевых слов оформляется в виде файла с расширением *.keu. На начальной стадии проведения экспертизы файл ключевых слов вносится в базу ключевых слов. После выбора команды «Сравнить», программа начинает процесс анализа программы обучения дисциплины на соответствие, используя метод сравнения ключевых слов. Каждый введенный файл ключевых слов сравнивается с уже имеющимися в базе ключевыми словами.

Взаимосвязь основной и вспомогательной программы можно представить в виде схемы 1 .

Надо отметить, что программа предусматривает два варианта создания файла ключевых слов:

1) ввод ключевых слов непосредственно в окне программы, где счетчик показывает количество введенных пользователем ключевых слов (30 ключевых слов);

2) набор ключевых слов в программе Блокнот в столбик (через Enter) с последующим сохранением файла с расширением *.keu.

Алгоритм формирования ключевого файла можно представить в виде блок-схемы 2.

Количество ключевых слов в программе строго ограничено, составляет 30 ключевых слов для каждой дисциплины. Данное ограничение связано с тем, что при составлении учебнометодического комплекса преподавательразработчик в зависимости от выделенных на дисциплину кредитов составляет программу обучения по дисциплине, в которой строго разграничены количество лекционных, практических, семинарских занятий. Это около 15 лекционных занятий для дисциплины. На каждое лекционное занятие формируется одно или два ключевых слова, которые должны четко передать содержание, основную мысль лекционного занятия. 


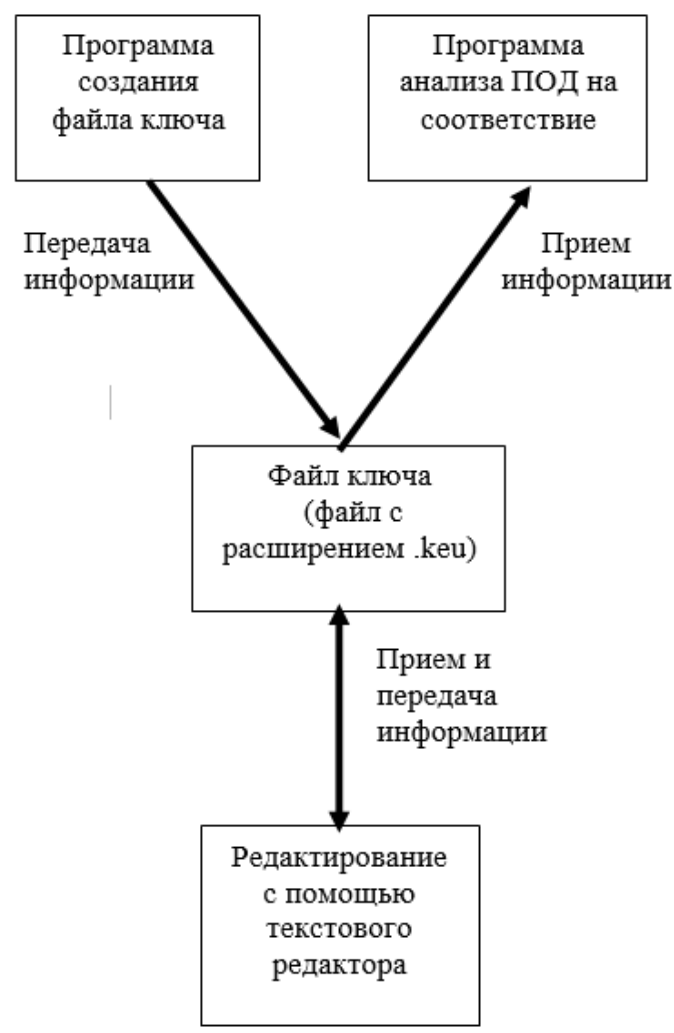

Рисунок 1 - Взаимосвязь основной и вспомогательной программы.
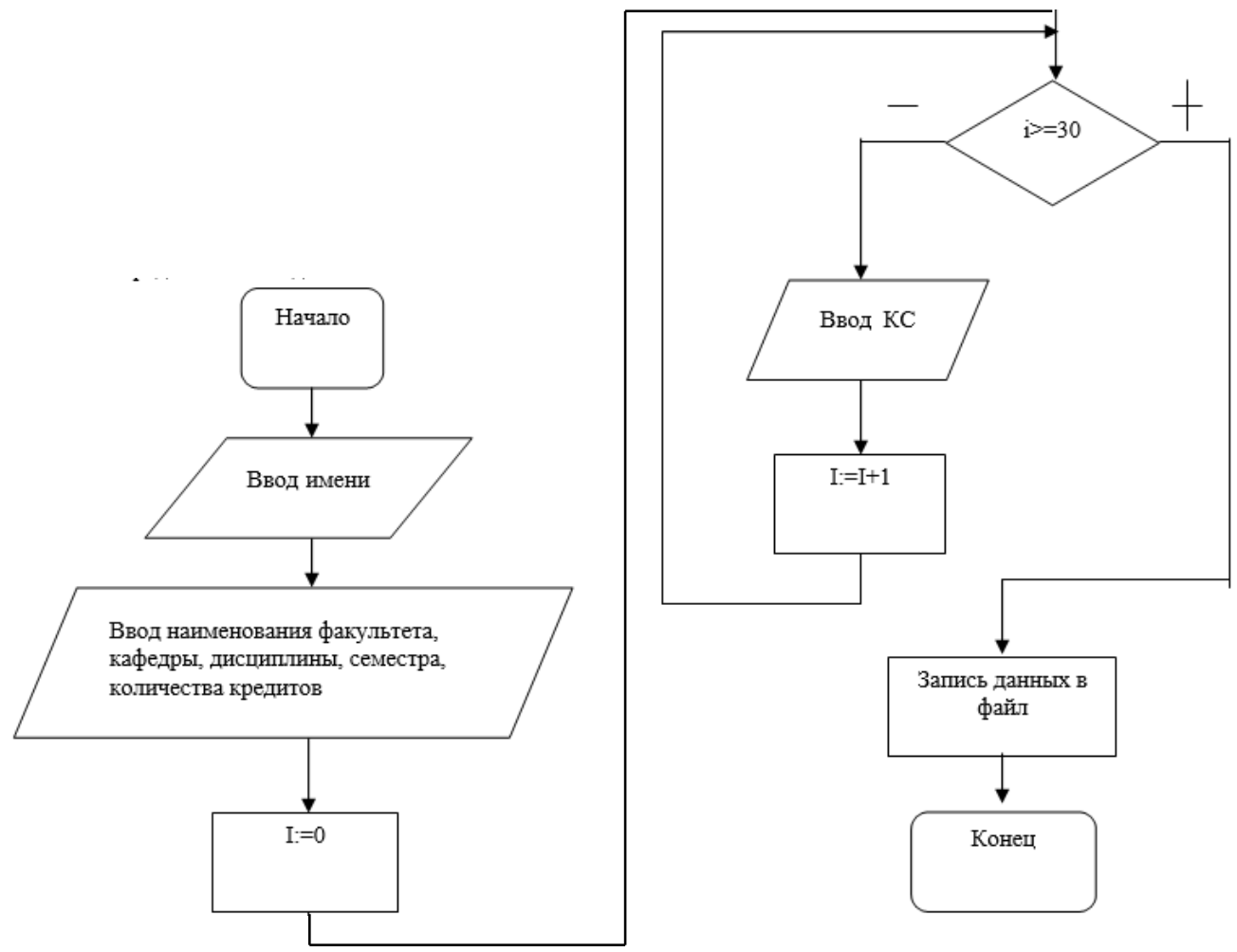

Рисунок 2 - Алгоритм формирования ключевого файла. 
В случае необходимости количество вводимых в программу ключевых слов может быть изменено до 40, 50 и т. д.

Возможны случаи, когда в качестве ключевого слова выступает словосочетание (материнская плата, оперативная память). В этом случае в программе учтен ввод ключевых слов словосочетаний через нижнюю черту (материнская_плата, оперативная_память). Пробел между двумя словами воспринимается программой как разделитель двух слов, а нижняя черта воспринимается как часть слова, т.е. разделенные нижней чертой два слова воспринимаются программой как одно единое слово.

Процесс экспертизы программы обучения дисциплины состоит в сравнении ключевых слов для учебно-методического комплекса дисциплин с уже имеющимися в базе ключевыми словами. Алгоритм сравнения ключевых слов с базой имеет следующий вид:

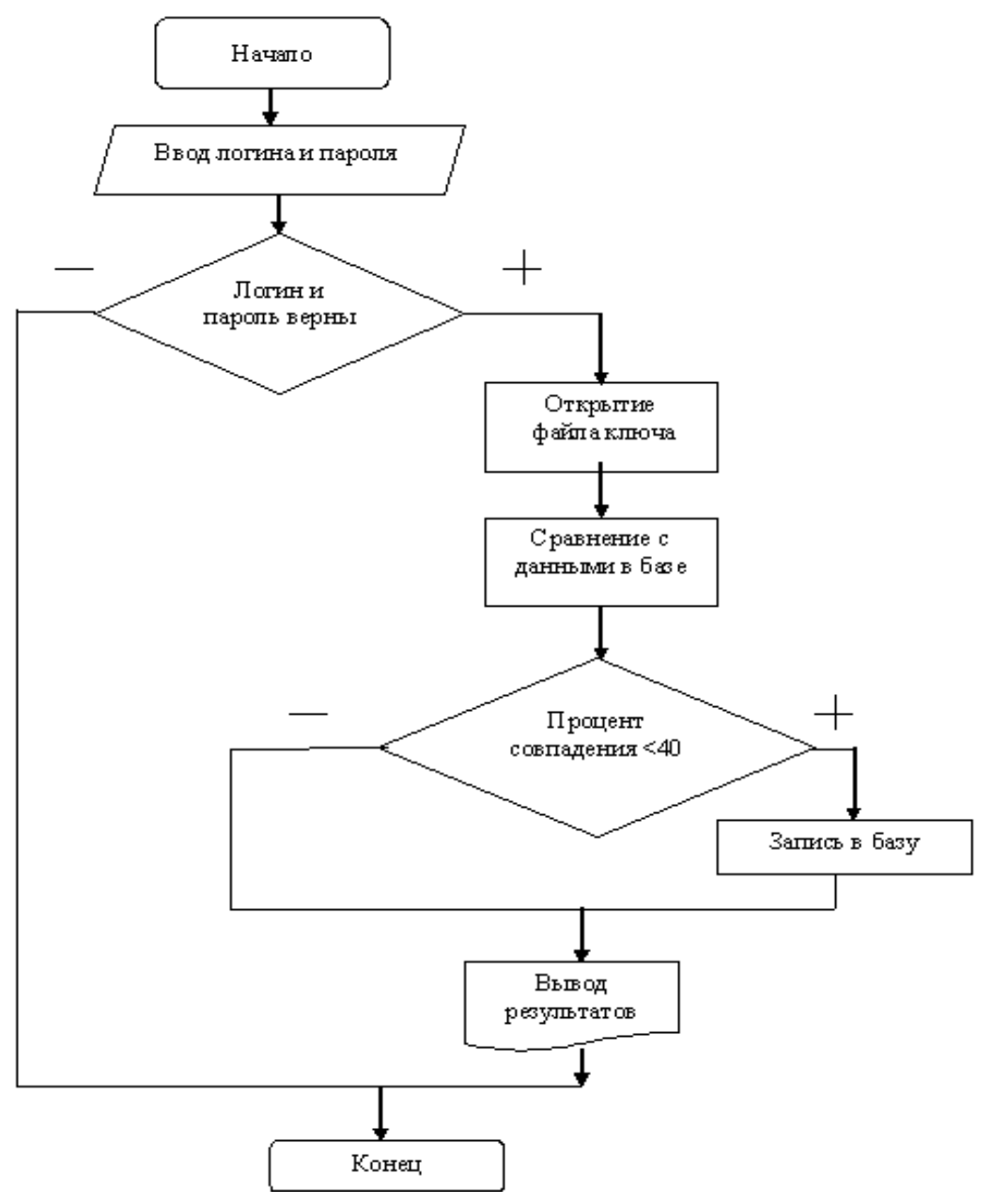

Рисунок 3 - Алгоритм сравнения ключевых слов с базой.

Каждая вводимая база ключевых слов по дисциплине автоматически сравнивается с уже занесенными в базу ключевыми словами. Если при сравнении совпадение ключевых слов составляет $40 \%$ и более, программа выдает сообщение о том, что учебная программа дублируется и, следовательно, данная ПОД дисциплины считается не прошедшей экспертизу и отправляется на переработку. Выходная информация выдается в виде сообщения о дублируемости программы обучения.
Каждая программа адаптирована к сложившимся в определенной сфере деятельности условиям экономики, управления и пользования, имеет определенный круг пользователей и примерно одинаковые с другими программами принципы работы, исходные данные, выходные формы, методики анализа результирующих показателей эффективности проекта.

Описанный программный продукт, как и любая автоматизированная система, обладает однотипной функциональной структурой: 
1) Источники информации. В роли источника информации выступают файлы ключевых слов учебная программа дисциплины и словарь синонимов.

2) Выполняет предварительную обработку данных (проверку и уточнение), а затем передает ее в базу данных или непосредственно для последующей обработки и анализа.

3) База данных. Данные являются результатом сбора информации, измерений характеристик объектов и процессов управления и в таких системах представляются в соответствии с определенными стандартами, образуя базу данных.

4) Обработка и анализ информации центральный блок автоматизированной системы. Он решает следующие задачи:

- управления базой данных, в том числе обеспечения ее обновления и целостности, защиты от несанкционированного доступа;

- реагирования в непредвиденных ситуациях, требующих быстрого решения;

- выполнение математических, статистических расчетов.

Программа автоматизации экспертизы программы обучения дисциплины позволяет в автоматическом режиме:

- формировать файл ключевых слов;

-

- дополнять словарь синонимов;

- $\quad$ контролировать ввод ключевых слов;

- пополнять базу ключевых слов;
- проводить сравнение ключевых слов;

- выполнять расчет процентного соотношения ключевых слов.

Разработанный программный продукт представляет собой программу, которая формирует базу данных и соответствующее приложение для автоматизации экспертизы программы обучения дисциплины, обладает достаточно широкими возможностями для изменения входных данных. Программный продукт ориентирован на конечного пользователя, не обладающего высокой квалификацией в области вычислительной техники, обладает простым, удобным, легко осваиваемым интерфейсом, который предоставляет конечному пользователю все необходимые для работы функции и в то же время не дает ему возможность выполнять какиелибо лишние действия. Окно программы содержит компоненты визуализирующие содержимое базы данных, кнопки, с помощью которых можно добавлять, изменять, удалять записи в базе данных.

Благодаря автоматизации процесс экспертизы программы обучения дисциплины существенно ускоряется, значительно повышается достоверность информации. Появляется возможность контроля за содержанием программы обучения дисциплины из блока по выбору, многие из которых дублируют уже имеющиеся программы обучения.

\section{References:}

1. Bidaybekov EY, Grigoriev SG, Grinshkun VV (2002) Data integration and analysis of educational development in the field of elearning. // Monograph. / Alma-Ata: MES RK, ASU. Abay. - 2002, 100 p.

2. Bidaybekov EY, Grinshkun VV (2001) Theoretical and technological approach to the development of e-learning. // Herald of ASU. Abay. Physical and mathematical series. AlmaAta. - 2001. № 2 (4). -pp. 32 - 37.

3. Emelyanova NC (2005) "Fundamentals of automated information systems: the manual" / NZ Emelyanov, T. Partyka, II Popov - M .: Forum: Infra-M, 2005. - 412 p.

4. Palchevsky BP, Friedman LS, Seleznev IF (1998) Educational-methodical complex learning tools. Part 1: Theoretical Foundations. - Mn., 1998.-120 p.

5. Rudakov AV (2005) Technology Software Development: A Handbook. allowance. Moscow: Publishing center "Academy", 2005.
6. Akulaeva OV (2007) Design of training and methodological support of innovative educational program modules Text $\therefore$ handbook / OV Akulaeva [et al.]; a. Ed. SA Goncharova. SPb .: RSPU. AI Herzen, 2007. - 159 p.

7. Linkov V, Linkov AV (2003) Questions of automation of management of educational process in high school. - ITO-2003.

8. Khomonenko AD, Tsygankov VM, Maltsev MG (2000) Database. SPb.: CORONA print 2000.

9. Hetagurov JA (2002) Fundamentals of automated systems for information processing and management (ASOIU): Textbook. Moscow: MEPhI, 2002. -252 p.

10. Norenkov IP (2002) Basics of computer-aided design: Textbook. For universities. 2nd ed., Rev. and add. Moscow: Publishing House of the MSTU. NE Bauman, 2002. - 336 p. 